\title{
General Lossy External Cloak with New Structures
}

\author{
Ensieh Ghasemi Mizuji ${ }^{1}$, Fatemeh Aghamohammadi ${ }^{1}$, Ali Abdolali ${ }^{1}$ \\ ${ }^{1}$ School of Electrical Engineering, Iran University of Science and Technology, Tehran, Iran \\ *corresponding author, E-mail: ensiyeh.ghasemi@ee.iust.ac.ir
}

\begin{abstract}
In this article we consider loss for all parts of external cloaks that are achieved by the different transformation functions. Each cloak has a different threshold for loss. We compare the simulation results with each other to find the best function for external cloaking.
\end{abstract}

\section{Introduction}

Controlling the electromagnetic waves for cloaking an object is an interesting field, which has recently received much attention[1-5]. Firstly, Pendry was capable to achieve a new method for controlling the electromagnetic waves with coordinate transformation [6-7]. All functions that satisfy transform conditions may be used [8]. One method to achieve the invisibility cloak is using the coordinate transformation. A new kind of cloaking based on this method is external cloak which make invisible an object in special distance out of the cloak [9-12]. The main idea for this design is a concept of complementary media [9, 13-16]. Complementary media is a material with negative refraction index which cancels the optical effects of any external object placed in a specific distance [17-20].

In this article, we consider several new coordinate transformation functions in linear, exponential and square forms for designing external cloak. Most of the works in this field especially in external cloak consider lossless media for cloak, but all the materials naturally are lossy, thus lossy environments were considered for inner core and outer shell of the cloaks and the simulation results were compared.

The results indicate that the acceptable loss of that inner core or outer shell of cloak to be capable to cloak the external region depends on the function of coordinate transformation that was used.

\section{Formulation and the structures}

The purpose of external cloak is cloaking the effects of an object that lies out of the cloak[7]. In this section we propose new functions for transformation. The simulation results are shown in figure 2.

According to the theory of coordinate transformation when we map one space to another one, the permittivity $\left(\varepsilon^{\prime}\right)$ and permeability $\left(\mu^{\prime}\right)$ of the transformed space $\left(r^{\prime}\right)$ are obtained from the following formulas:

$$
\varepsilon^{\prime}=A \varepsilon A^{T} / \operatorname{det}(A)
$$

$$
\mu^{\prime}=A \mu A^{T} / \operatorname{det}(A)
$$

Where, $\varepsilon$ and $\mu$ are the permittivity and permeability of old space $(r)$ respectively. A is the Jacobian of transformation matrix and the components are obtained by $A_{i j}=\partial x_{i}^{\prime} / \partial x_{j}[16,21]$. Complementary medium is the material with negative permittivity and permeability of the outer space which have the special property that if an object is placed between 0 to $\mathrm{L}$ it cancels the optical effect as there is nothing[15]. (Figure 1)

The main idea to achieve material parameters is combining the theory of complementary media and coordinate transformation. The first step is transformation of the region $a<r<b$ on to the region $b<r^{\prime}<c$, this transformation result $\mathrm{a}<\mathrm{r}<\mathrm{b}$ with negative refractive index so the region $a<r^{\prime \prime}<c$ is optically void[7-9]. Any transformation function that satisfies these transformation conditions is acceptable[6].
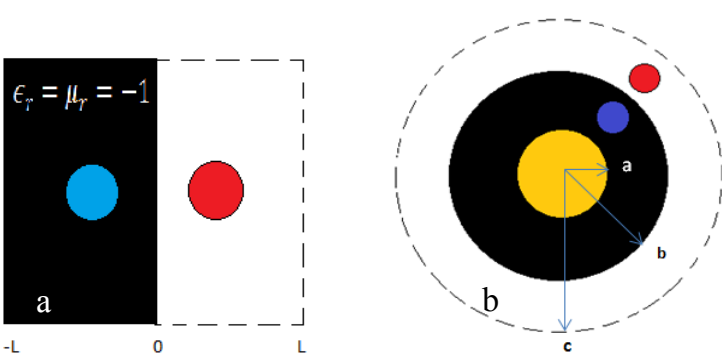

Figure 1. (a) a slab of complementary medium which makes the external object invisible by canceling the optical effects of it. (b) a cylindrical form complementary medium which has the same effect as (a).

Hence we use three functions in order to transform $a<r<b$ to $b<r^{\prime}<c$ as:

$$
\begin{aligned}
& f\left(r^{\prime}\right)=\left[\left(r^{\prime}-b\right)(c-b) /(a-b)\right]+b \\
& f\left(r^{\prime}\right)=\left(e^{-r^{\prime}} / e^{-a}\right)\left[\left(r^{\prime}-b\right)(c-b) /(a-b)\right] b
\end{aligned}
$$




$$
f\left(r^{\prime}\right)=\left(\left(r^{\prime}-b\right) /(a-b)\right)^{2}(c-b) \quad \#
$$

Therefore the permittivity $\left(\varepsilon^{\prime}\right)$ and permeability $\left(\mu^{\prime}\right)$ are obtained by:

$$
\begin{aligned}
& \varepsilon_{r}^{\prime}=\mu_{r}^{\prime}=\left(f\left(r^{\prime}\right) / r^{\prime}\right)\left(1 / f^{\prime}\left(r^{\prime}\right)\right) \\
& \varepsilon_{\theta}^{\prime}=\mu_{\theta}^{\prime}=\left(r^{\prime} / f\left(r^{\prime}\right)\right) f^{\prime}\left(r^{\prime}\right) \\
& \varepsilon_{z}^{\prime}=\mu_{z}^{\prime}=\left(f\left(r^{\prime}\right) / r^{\prime}\right) f^{\prime}\left(r^{\prime}\right)
\end{aligned}
$$

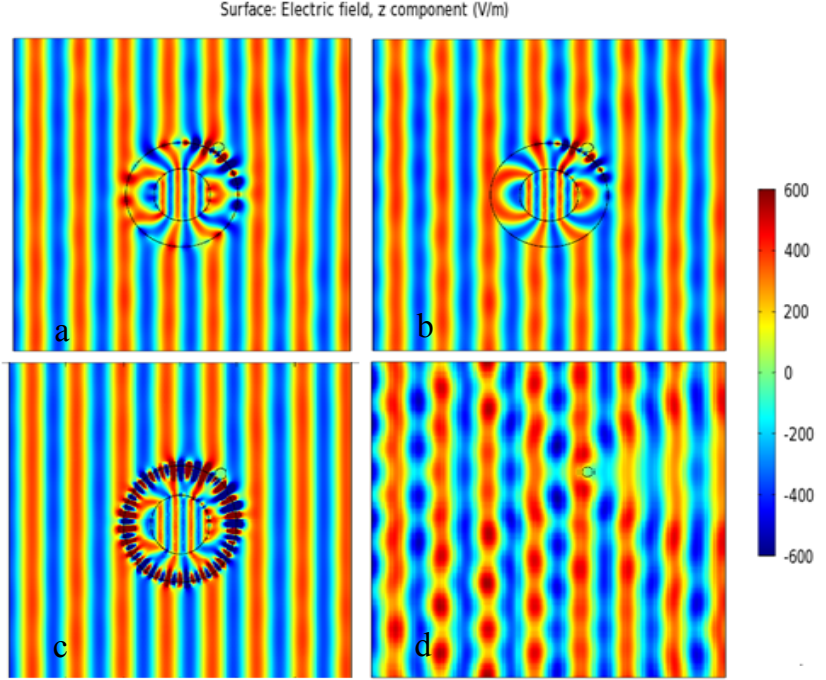

Figure 2. simulation results for a)linear function .b)exponential function. c)square function. d)without cloak

And the transformation function $\left(r^{\prime \prime}=r^{\prime}(a / c)\right)$ transforms 0 $<r<a$ to $0<r^{\prime \prime}<c$. The $\varepsilon^{\prime \prime}$ and $\mu^{\prime \prime}$ are obtained same as $\varepsilon^{\prime}$ and $\mu^{\prime}$. Figure 2 shows the simulation results. Geometry parameters used in the simulations are chosen as $a=0.1 \mathrm{~m}$, $b=0.2 \mathrm{~m}$ and $c=0.3 \mathrm{~m}$. scattering pattern of the object with $\varepsilon_{r}=-2$ and $\mu_{r}=1$ in figure $2-\mathrm{d}$ has been shown. The object is hidden by the cloaks that are transformed with functions which are derived from equations 3-5.

\section{Investigation of lossy structures}

Lossy environments are considered for the structures which their permeabilities and permittivities are obtained from transformation functions 3 and 4 and 5 by substituting in equations 6 and 7 and 8. First we loss for inner core is cosidered. Simulation results are shown in figures 3-6.

Figure 3 shows the simulation result for $\sigma=.01$. The object is still cloak therefore it is realized that none of the structures is sensitive to low amount of loss. In figure 4 we increase $\sigma$ to .04 . By comparing the images we find out that the only cloak structure is transformed with square function. So the results show that the square transformation function has slightly better performance than others and linear is better than exponential.

Then we consider loss for outer shell. We expect the cloak performance to be different. Figures 5 and 6 show the simulation results.

Since outer shell is more sensitive than inner core, it has lower threshold of loss amplitude to be cloak. Figure 5 shows the simulation result for $\sigma=.0008$ in different structures it cloud be observe that all the structures are cloak. By increasing the amount of loss to $\sigma=.001$ it shows the cloak that is transformed with exponential function is still cloak but other structures are not cloak. Therefore linear and exponential transformation function has better performance but exponential function is slightly better than linear. To confirm our conclusion we draw $\tan (\delta)$ as the function of $r$ (figures 7-9).

Figure 7 shows that the exponential function has the smallest $\tan (\delta)$ and linear function has smaller $\tan (\delta)$ than the square function.so results verified our conclusion.

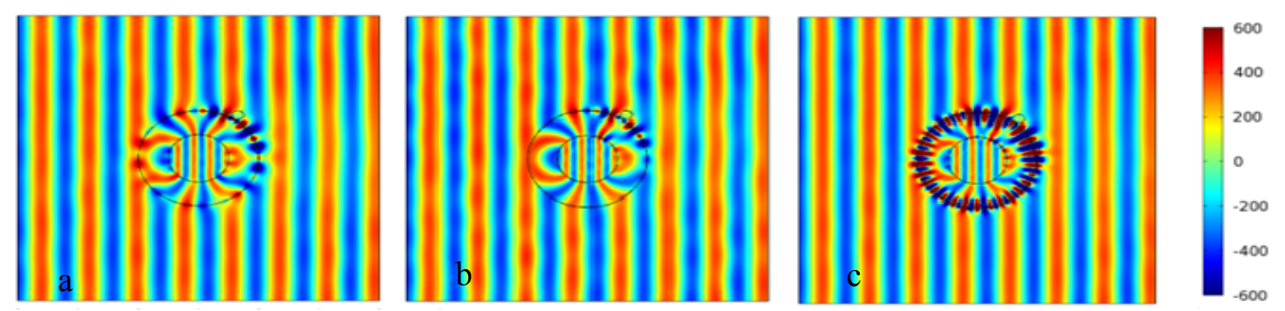

Figure 3. simulation result with $\sigma=.01$ for inner core a) linear function b)exponential function c)square function.
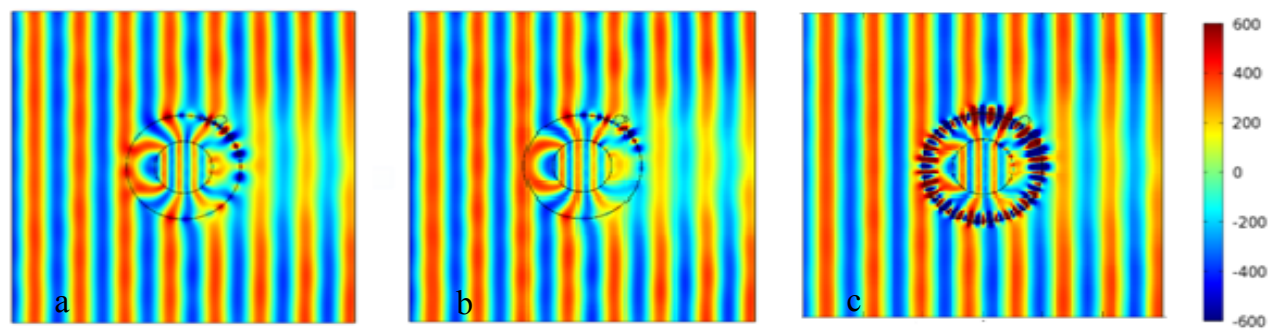

Figure 4. simulation result with $\sigma=.04$ for inner core a) linear function b)exponential function c)square function. 


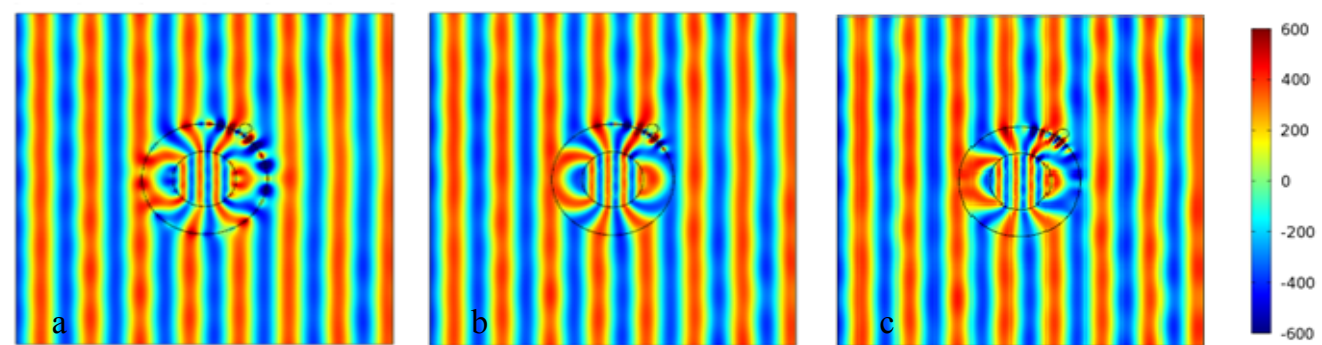

Figure 5: simulation result with $\sigma=.0008$ for outer shell a) linear function b)exponential function c)square function.

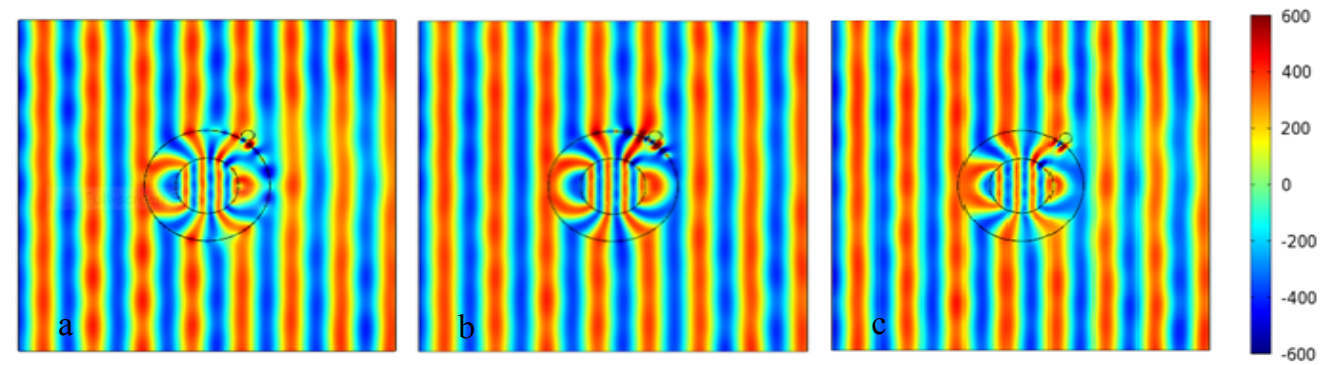

Figure 6: simulation result with $\sigma=.001$ for outer shell a) linear function b)exponential function c)square function.

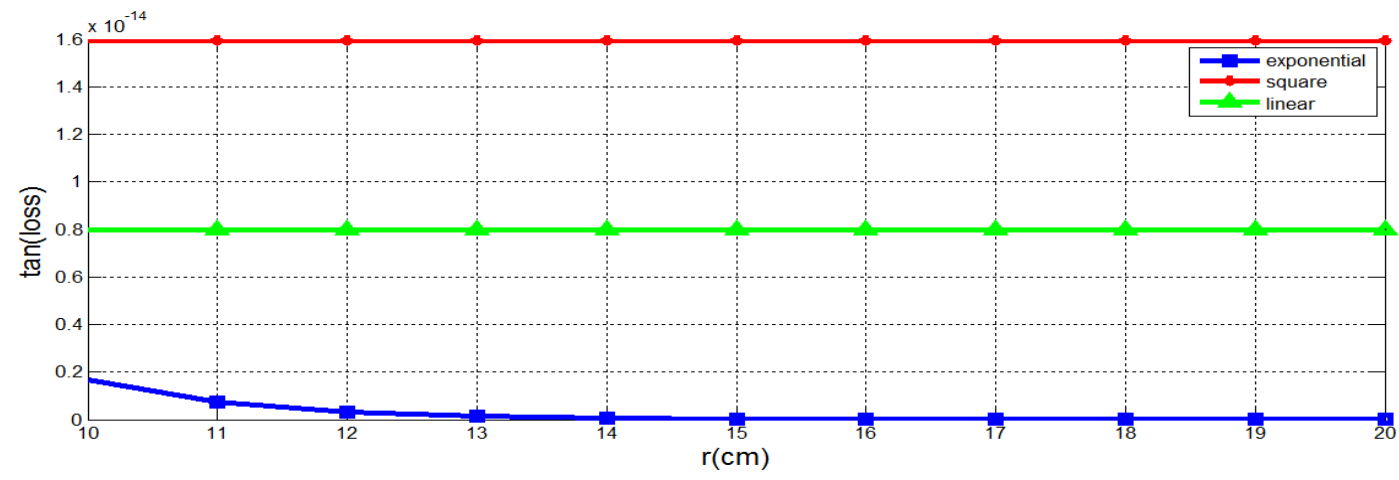

Figure 7: r component of $\tan (\delta)$, linear transformation, exponential transformation and square transformation are drown.

\section{Conclusions}

Different external cloaks are designed with different transformation functions which satisfy the boundary condition purposes and considered loss for all parts. Results show that it is possible to improve the cloak efficiency with varying the coordinate transformation function. In this paper cloaks with linear, exponential and square forms are simulated. With comparing simulation results it is found out linear and exponential forms are better than square function due to have lower loss tangent. The exponential form is the best and has better efficiency than others.

\section{References}

[1] B.Zhang, H. Chen, and B.-I. Wu," Practical Limitations of An Invisibility Cloak",Progress In Electromagnetic Research,407416,(2009).

[2] Q.Cheng, W. X. Jiang, and T.-J. Cui," Investigations of The Electromagnetic Properties of Three-Dimentinal ArbitrarilyShaped Cloaks", Progress In Electromagnetic Research,105117,(2009).
[3] X.Chen," Implicit Boundary Conditions in TransformationOptics Cloaking for Electromagneticwaves"Progress In Electromagnetic Research,521-534,(2011).

[4] K.Agarwal, X. Chen, L. Hu, H. Liu, and G. Uhlmann," Polarization-Invariant Directional Cloaking by Transformation Optics”,Progress In Electromagnetic Research, 415-423,(2011).

[5] S.Y.Lai, H.Chen, Zhao-Qing Zhang, and C. T. Chan, "Complementary Media Invisibility Cloak that Cloaks Objects at a Distance Outside the Cloaking" physical review letters,102, 093901 (2009).

[6] J.B.Pendry, D. Schurig, and D. R. Smith, "Controlling Electromagnetic Fields," Science 312, 1780-1782 (2006).

[7] U.Leonhardt, "Optical Conformal Mapping," Science 312, 1777-1780 (2006).

[8] Y.Luo, H. Chen, J. Zhang, L. Ran, and J. A. Kong "Design and Analytically Full-wave Validation of the Invisibility Cloaks, Concentrators, and Field Rotators Created With a General Class of Transformations," Physics (2007).

[9] Y.Lai, H. Chen, Z. Zhang, and C. T. Chan, "Complementary Media Invisibility Cloak that Cloaks Objects at a Distance Outside the Cloaking Shell," Physical Review Letters, 102, 093901 (2009).

[10] T.Philbin," Cloaking At a Distance", Physics 2, 17 (2009). 
[11] C.F.Yang, M. Huang, J. J. Yang, and Z. Xiao, "An External Cloak With Arbitrary Cross Section Based On Complementary Medium," Progress In Electromagnetics Research M, Vol. 10, 1324, (2009).

[12] Y.Lai, H. Chen, Z. Zhang, and C. T. Chan, "External Invisibility Device That Cloaks Objects At a Distance," SPIE Newsroom, DOI:10.1117/2.1200907.1720 (2009).

[13] J.B.Pendry, "Negative Refraction Makes a Perfect Lens," Phys. Rev. Lett. 85, 3966 -3969 (2000).

[14] J.B.Pendry and S.A. Ramakrishna, "Focusing Light Using Negative Refraction," Journal of Physics-Condensed Matter, 15, 6345-6364 (2003)

[15] K.Kobayashi, " Complementary Media of Electrons," J. Phys. Condens. Matter 18, 3703-3720 (2006).

[16] W.Yan, M. Yan, and M. Qiu," Necessary and Sufficient Conditions for Reflectionless Transformation Media in An Isotropic and Homogenous Background," arXiv, 0806.3231(2008).

[17] G.W.Milton, and N. A. P. Nicorovici, "On the Cloaking Effects Associated With Anomalous Localized Resonance," Proc. R. Soc. London, Ser. A 462, 3027-3059 (2006).

[18] N.A.P.Nicorovici, G.W. Milton, R.C. McPhedran, L.C. Botten, "Quasistatic Cloaking of Two-Dimensional Polarizable Discrete Systems by Anomalous Resonance," Opt. Express 15, 6314-6323 (2007).

[19] G.W. Milton, N. P. Nicorovici, R. C. McPhedran, K. Cherednichenko, and Z.Jacob, "Solutions in Folded Geometries, and Associated Cloaking Due to Anomalous Resonance," arXiv,0804.3903(2008).

[20] O. P.Bruno and S. Lintner, "Superlens-Cloaking of Small Dielectric Bodies In the Quasistatic Regime," J. Appl. Phys. 102(12), 124502 (2007).

[21] A. V Kildishev, and E. E. Narimanov, "Impedance-Matched Hyperlens," Opt. Lett. 32(23), 3432-3434 (2007). 